\title{
Provider and Patient-Related Barriers to and Facilitators of Digital Health Adoption for Hypertension Management: Review
}

\author{
Ramya Palacholla ${ }^{1,2}$, MPH; Nils Fischer ${ }^{1,2}$, MPH; Amanda Coleman ${ }^{3}$, MPH; Stephen Agboola ${ }^{1,2}$, MD, MPH; Jennifer \\ Felsted $^{1,2}$, PhD; Kate Kirley ${ }^{1,2}$, MD, MPH; Chelsea Katz ${ }^{1,2}$, PHD; Stacy Lloyd ${ }^{3}$, PhD; Kamal Jethwani ${ }^{1,2}$, MD, MPH \\ ${ }^{1}$ Partners Connected Health Innovation, Boston, MA, United States \\ ${ }^{2}$ Harvard Medical School, Boston, MA, United States \\ ${ }^{3}$ American Medical Association, Chicago, MA, United States
}

\section{Corresponding Author:}

Ramya Palacholla, MPH

Partners Connected Health Innovation

25 New Chardon

Suite 300

Boston, MA,

United States

Phone: 9199779

Email: rpalacholla@mgh.harvard.edu

\begin{abstract}
Background: Management of hypertension employing digital health technologies (DHT) has been proven to improve long-term patient outcomes. However, the uptake of DHT has been surprisingly low in clinical practice. Despite showing great promise to improve patient outcomes and disease management, there is limited information on the factors that contribute to the limited adoption of DHT particularly for hypertension management.
\end{abstract}

Objective: This review provides a comprehensive summary of barriers to and facilitators of DHT adoption for hypertension management reported in the published literature with a focus on provider and patient-related perspectives.

Methods: This review was conducted using the methodological framework developed by Arskey and O'Malley. Systematic literature searches were conducted on PubMed/ Medline, CINAHL, and EMBASE. Articles that reported on barriers to and/or facilitators of digital health adoption for hypertension management, published in English between 2008 and 2017 were eligible. Studies not reporting on barriers or facilitators to DHT adoption for management of hypertension were excluded. A total of 2299 articles were identified based on criteria above after removing duplicates and were assessed for eligibility. Of these, 2165 references did not meet the inclusion criteria. After assessing 134 studies in full-text, 98 studies were excluded (full texts were unavailable or studies did not fulfill the inclusion criteria) resulting in a final set of 32 articles. Four hand-picked articles were also included in the review.

Results: A total of 36 studies were selected for data extraction after abstract and full-text screening by two independent reviewers. All conflicts were resolved by a third reviewer. Thematic analysis was conducted to identify major themes pertaining to barriers and facilitators of DHT from both provider and patient perspectives. Key facilitators of DHT adoption by physicians identified include integration with clinical workflow, ease of use, improvement in patient outcomes and organizational support. Improved patient-provider relationship, positive impact on well-being and self-management were most frequently reported facilitators for patients. Barriers to use of DHTs reported by physicians include mistrust in technology, data security, lack of usability, and organizational support and commitment to DHT adoption. Finally, a lack of perceived benefit from technology, lack of ease of use and concern over data security were some of the barriers commonly reported by patients.

Conclusions: Although technology has evolved at a rapid pace, many facilitators and barriers reported by patients and providers are consistent over time. Our findings suggest the settings and context in which DHT are implemented, and individuals involved in implementation such as providers, patients and leadership influence adoption in healthcare settings. Real-world testing and incorporating feedback from key stakeholders including patients, providers and hospital management while designing DHT will improve their usability and thereby the adoption. Finally, to fully realize the potential of digitally enabled hypertension management, there is a greater need to validate these technologies to provide patients and providers with reliable and accurate information on both clinical outcomes and cost effectiveness. 
(iproc 2018;4(2):e11904) doi: 10.2196/11904

\section{KEYWORDS}

barriers; health; digital; patient; provider

Edited by T Hale; this is a non-peer-reviewed article. Submitted 09.08.18; accepted 29.08.18; published 17.09.18.

Please cite as:

Palacholla R, Fischer N, Coleman A, Agboola S, Felsted J, Kirley K, Katz C, Lloyd S, Jethwani K

Provider and Patient-Related Barriers to and Facilitators of Digital Health Adoption for Hypertension Management: Review

iproc 2018;4(2):e11904

URL: http://www.iproc.org/2018/2/e11904/

doi: $10.2196 / 11904$

PMID:

(CRamya Palacholla, Nils Fischer, Amanda Coleman, Stephen Agboola, Jennifer Felsted, Kate Kirley, Chelsea Katz, Stacy Lloyd, Kamal Jethwani. Originally published in Iproceedings (http://www.iproc.org), 17.09.2018. This is an open-access article distributed under the terms of the Creative Commons Attribution License (https://creativecommons.org/licenses/by/4.0/), which permits unrestricted use, distribution, and reproduction in any medium, provided the original work, first published in Iproceedings, is properly cited. The complete bibliographic information, a link to the original publication on http://www.iproc.org/, as well as this copyright and license information must be included. 